\title{
Special Issue: Papers by Plenary and Invited Lecturers at the 24th International Symposium on Plasma Chemistry (ISPC 24), 9-14 June 2019, Naples, Italy
}

\section{Matteo Gherardi ${ }^{1,2}$}

Published online: 22 April 2020

(c) Springer Science+Business Media, LLC, part of Springer Nature 2020

The 24th International Symposium on Plasma Chemistry (ISPC 24) was held in the beautiful city of Naples, Italy at the Conference Center Terminal in the waterfront district. The conference was chaired by Prof. Vittorio Colombo from Alma Mater Studiorum-University of Bologna and co-chaired by Prof. Pietro Favia from the University of Bari "Aldo Moro" and Dr Matteo Gherardi from Alma Mater Studiorum-University of Bologna.

ISPC 24 welcomed 563 attendees, including 182 students, from 43 countries. A total of 570 oral and poster presentations were delivered, including the Plasma Chemistry Award lecture, 5 Plenary Speaker lectures, 22 Invited Speaker lectures, 139 regular oral presentations, 375 posters and 283 -min poster pitches (all by students), distributed over the 10 scientific and application topics. The 2-day Summer School preceding the symposium welcomed 52 attendees and 15 speakers. To foster the participation of graduate students and researchers from developing or under-represented countries, the International Plasma Chemistry Society (IPCS) sponsored 30 early-bird registrations.

The Plasma Chemistry Award 2019, the highest recognition bestowed by the International Plasma Chemistry Society (IPCS) for lifetime achievements in the field of Plasma Chemistry, was awarded to Professor Alexander Fridman from Drexel University. Dr Milan Hrabovsky from the Academy of Sciences of the Czech Republic, Dr John Lowke from CSIRO, Australia and Prof. Colombo from Alma Mater Studiorum-University of Bologna, Italy were elevated to the rank of IPCS Fellow (FIPCS).

The Young Investigator Awards 2019 were given to Xuekai Pei (1st place) from the University of California, Berkeley, USA and Augusto Stancampiano (2nd place) from GREMI, France. Oral presentation awards were given to Aric Rousso (1st place) from Princeton University, USA, Jiri Kratochvil (2nd place) from Charles University, Czech Republic and Zhaohan Li (3rd place) from the University of Minnesota, USA. Poster presentation awards were given to Richard Cimerman from Comenius University, Slovakia, Ezgi Bulbul from ETH Zurich and EMPA, Switzerland and Toshisato Ono from the University of Minnesota,

Matteo Gherardi

matteo.gherardi4@unibo.it

1 Department of Industrial Engineering, Alma Mater Studiorum-Università di Bologna, Bologna, Italy

2 Interdepartmental Center for Industrial Research- Advanced Applications in Mechanical Engineering and Materials Technology, Alma Mater Studiorum-Università di Bologna, Bologna, Italy 
USA. 3-min poster pitch awards were given to Ana Sofia Morillo-Candas from Ecole Polytechnique, Palaiseau, France and Katerina Polaskova from Masaryk University, Czech Republic. These awards were sponsored by Journal of Physics D: Applied Physics, MDPI, Clinical Plasma Medicine, Plasma Chemistry and Plasma Processing (Springer) and Plasma Processes and Polymers (Wiley), which provided the certificates, monetary awards and book vouchers. Thematic awards for material processing, consisting of a certificate and a monetary award, were provided by Biointerphases and were assigned to Charlotte Ruhmlieb (best oral) from the University of Hamburg, Germany and Rouba Ghobeira (best poster) from Ghent University, Belgium. Thematic awards for thermal plasmas, consisting of a certificate and a monetary award, were provided by the Journal of Thermal Spray Technology and were assigned to Chayma Mohsni Ruhmlieb (best oral) from the National School of Engineering of Monastir, Tunisia and the Leibniz Institute for Plasma Science and Technology, Germany, and Hiroki Munekata (best poster) from Kyushu University, Japan.

Numerous sponsors contributed to the symposium, including Alma Mater StudiorumUniversity of Bologna, the University of Bari Aldo Moro, AlmaPlasma, Plasma Matters, Gambetti, Kapteos, Nadir, Plasmapps, Plasmionique, Skintech Laboratory, Hypertherm, Journal of Physics D: Applied Physics, ASM International, Wiley, Springer, Clinical Plasma Medicine, AVS and MDPI. Several of these sponsors had exhibition booths during the symposium.

This special issue of Plasma Chemistry and Plasma Processing contains eight manuscripts based on the invited contributions presented at ISPC 24. All papers submitted underwent the full refereeing procedure of the journal. The peer-reviewed articles are by plenary speakers Achim von Keudell (Pattern formation in high power impulse magnetron sputtering (HiPIMS) plasmas) and Anne Bourdon (Numerical study of jet-target interaction: influence of dielectric permittivity on the electric field experienced by the target) and from invited speakers Julia Bandow (A dielectric barrier discharge plasma degrades proteins to peptides by cleaving the peptide bond), Maria Adriana Creatore (Plasma-assisted ALD of highly conductive $\mathrm{HfN}_{\mathrm{x}}$ : on the effect of energetic ions on film microstructure), Vasco Guerra (Dynamics of gas heating in the afterglow of pulsed $\mathrm{CO}_{2}$ and $\mathrm{CO}_{2}-\mathrm{N}_{2}$ glow discharges at low pressure), Masaya Shigeta (Simulating turbulent thermal plasma flows for nanopowder fabrication), Juan Pablo Trelles (Nonequilibrium phenomena in (quasi-) thermal plasma flows), Zdenko Machala (Transient spark discharge generated in various $\mathrm{O}_{2} / \mathrm{N}_{2}$ gas mixtures: reactive species in the gas and water and their antibacterial effects) and their coauthors. This selection is representative of the general themes of the symposium, with contributions dealing with both non-thermal and thermal plasma science and technology by means of experimental and computational approaches. Complete Symposium proceedings are available online at http://www.ispc-conference.org/.

Finally, we wish to thank all who contributed to the organization of ISPC 24!

Publisher's Note Springer Nature remains neutral with regard to jurisdictional claims in published maps and institutional affiliations. 